\title{
ВЕРОЯТНОСТНЫЙ ПОДХОД К РЕШЕНИЮ ТРЕХИНДЕКСНОЙ АКСИАЛЬНОЙ ЗАДАЧИ О НАЗНАЧЕНИЯХ. РАЗВИТИЕ ПОИСКОВЫХ СПОСОБНОСТЕЙ
}

\author{
А. Г. Трегубов, С. Н. Медведев \\ Воронежский государственный университет
}

Поступила в редакцию 22.10.2018 г.

\begin{abstract}
Аннотация. В статье рассматривается адаптивный алгоритм решения трехиндексной аксиальной задачи о назначениях. Алгоритм основан на переходе к вероятностной постановке задачи. На основе вычислительного эксперимента рассмотрена возможность его улучшения. Для этого вводится понятие окрестности решения задачи на основе расстояния Хэмминга, и рассматривается переход из окрестности одной точки в окрестность другой на основе настройки параметров алгоритма. В результате предлагается изменение параметров алгоритма по некоторой функциональной зависимости от числа итераций. В завершении приводятся результаты вычислительного эксперимента и выводы.

Ключевые слова: трехиндексная аксиальная задача о назначениях, адаптивный алгоритм, вероятностная постановка, локальный поиск.

Annotation. The article considers the adaptive algorithm for solving axial 3-index assignment problem. The algorithm is based on the transition to a probabilistic statement of the problem. On the basis of a calculation experiment, the possibility of its improvement was considered. For this, the concept of a neighborhood of a solution based on the Hamming distance is introduced. As a result, the proposed change in the parameters of the algorithm for some function. Finally, the results of the computational experiment and conclusions are given.
\end{abstract}

Keywords: axial 3-index assignment problem, adaptive algorithm, probabilistic statement, local search.

\section{ВВЕДЕНИЕ}

Естественным обобщением классической задачи о назначениях $(3 \mathrm{OH})$ является трехиндексная аксиальная $3 \mathrm{OH}$, которая состоит в таком выборе $n$ элементов трехмерной матрицы затрат $\left(c_{i j}^{k}\right)$ размерности $n \times n \times n$, что в каждом ее сечении выбран ровно один элемент, и сумма всех выбранных элементов минимальна.

Для получения математической записи аксиальной $3 \mathrm{OH}$ вводится $n^{3}$ переменных вида:

где $i, j, k=\overline{1, n}$.

$$
x_{i j}^{k}=\left\{\begin{array}{l}
1, \\
0,
\end{array}\right.
$$

В результате задача принимает следующий вид:

$$
\sum_{i=1}^{n} \sum_{j=1}^{n} \sum_{k=1}^{n} c_{i j}^{k} x_{i j}^{k} \rightarrow \min
$$

( Трегубов А. Г., Медведев С. Н., 2018

$$
\begin{aligned}
& \sum_{j=1}^{n} \sum_{k=1}^{n} x_{i j}^{k}=1, i=\overline{1, n}, \\
& \sum_{i=1}^{n} \sum_{k=1}^{n} x_{i j}^{k}=1, \quad j=\overline{1, n}, \\
& \sum_{i=1}^{n} \sum_{j=1}^{n} x_{i j}^{k}=1, \quad k=\overline{1, n}, \\
& x_{i j}^{k}=\{0,1\}, i, j, k=\overline{1, n} .
\end{aligned}
$$

Данная задача является $N P$-трудной [4], что стимулирует разработку приближенных алгоритмов с оценками качества получаемых решений. Такого рода алгоритмам посвящено множество работ, некоторые из которых представлены далее.

В [10] рассматривается полный трехдольный граф, вершины которого соответствуют индексам $i, j$ и $k$ соответственно. Каждое его ребро $[i, j]$ (или $[i, k]$, или $[j, k])$ имеет длину $d(i, j)$ (или $d(i, k)$, или $d(j, k))$. Предлагаются две модели трехиндексной аксиальной 
задачи: в первой затраты $c_{i j}^{k}$ определяются как сумма трех длин $c_{i j}^{k}=d(i, j)+d(i, k)+d(j, k)$, а во второй - как сумма двух более коротких. Предлагаются $\varepsilon$-приближенные алгоритмы, которые получают решение, значение которого в первом случае не хуже, чем $3 / 2$ от оптимального значения, а во втором случае - чем $4 / 3$ от оптимального значения, показана $\mathrm{NP}$-трудность данной задачи.

В работе [3] представлен асимптотически точный подход к построению алгоритмов решения аксиальных задач большой размерности на случайных входах и получены условия, при которых эти алгоритмы позволяют находить асимптотически точные решения многоиндексной аксиальной $3 \mathrm{OH}$.

В [1] предложен эвристический алгоритм решения NP-трудных многоиндексных задач с декомпозиационной структурой (применимый в том числе и для аксиальной задачи о назначениях), степень отклонения от оптимума находимого решения ограничена через значение критерия вспомогательной задачи ЛП.

В работе [2] найдены достаточные условия сводимости к задаче поиска потока в сети, предложенный полиномиальный алгоритм в частности применим для решения аксиальной задачи о назначениях с критерием специальной декомпозиционной структуры.

Авторами статьи также предложен свой метод решения трехиндексной аксиальной 3ОН. Его основная идея и алгоритм представлены в работах $[7,8]$, а также будут кратко описаны ниже для лучшего понимания дальнейшего исследования. Рассматриваемый адаптивный алгоритм обладает как рядом достоинств, таких как гибкость настройки и возможность внедрения дополнительных эвристических приемов, так и некоторыми недостатками, основной из которых - сравнительно большое время работы. Целью данной работы является развитие поисковых возможностей алгоритма для улучшения получаемого значения целевой функции, что является возможным благодаря гибкости настройки его параметров.

\section{МАТЕРИАЛЫ И МЕТОДЫ}

\section{Адаптивный алгоритм}

Для трехиндексной аксиальной задачи известен базовый «жадный» алгоритм, основанный на поиске минимального элемента в плоских матрицах при фиксировании одного из индексов [4]. На его основе предлагается построение адаптивного алгоритма решения аксиальной трехиндексной $3 \mathrm{OH}$ (1)-(5). Для этого осуществляется переход к вероятностной постановке задачи на основе рандомизации переменных [5].

Задача минимизации целевой функции

$$
L(X) \rightarrow \min _{X \in \Omega}
$$

заменяется задачей минимизации ее математического ожидания

$$
M(L(\mathrm{X})) \rightarrow \min _{\{\mathrm{X}\}: X \in \Omega},
$$

где $\{\mathrm{X}\}$ - множество случайных величин $\mathrm{X}$ с реализациями $X$ из множества $\Omega$.

Здесь $\mathrm{X}=\left[\mathrm{X}^{1} \ldots \mathrm{X}^{n}\right]$ - кубическая матрица случайных величин,

$\mathrm{X}^{k}=\left[\mathrm{X}_{1}^{k} \ldots \mathrm{X}_{n}^{k}\right]^{T}-$ матрица $n \times n$ случайных величин,

$\mathrm{X}_{i}^{k}=\left(\mathrm{X}_{i 1}^{k} \ldots \mathrm{X}_{i n}^{k}\right)$ - строка матрицы $\mathrm{X}^{k}$,

$\mathrm{X}_{i j}^{k}$ - случайная величина с рядом распределения:

\begin{tabular}{|l|c|c|}
\hline Возможные значения & 1 & 0 \\
\hline Вероятность $p$ & $p_{i j}^{k}$ & $q_{i j}^{k}=1-p_{i j}^{k}$ \\
\hline
\end{tabular}

где $i, j, k=\overline{1, n}$.

Вводятся полные группы событий $A_{i j}^{k}$, для каждого $k=\overline{1, n}$, где $A_{i j}^{k}$ - событие, заключаю-

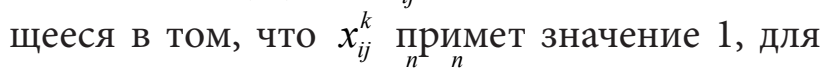
каждого $k=\overline{1, n}$, т. е. $\sum_{i=1}^{n} \sum_{j=1}^{n} p_{i j}^{k}=1, k=\overline{1, n}$.

Подробное описание этапов разработки алгоритма приводится в [8]. Здесь же приведем главное отличие алгоритма от базового «жадного», которое заключается в следующем:

при фиксированном $k$ вместо

$$
\begin{aligned}
& \min _{i, j} c_{i j}^{k}=c_{\mu \nu}^{k} \\
& \text { въцчислить } \\
& \min _{i, j}\left\{c_{i j}^{k}-\sum_{l=k+1}^{n} \sum_{j=1}^{n} c_{i j}^{l}\left(p_{i j}^{l}\right)^{N}+\sum_{l=k+1}^{n} \sum_{i=1}^{n} c_{i j}^{l}\left(p_{i j}^{l}\right)^{N}\right\} .
\end{aligned}
$$


Вероятностный подход крешению трехиндексной аксиальной задачи о назначениях ...

То есть вместо выбора минимального элемента в плоской матрице, выбираются минимальные возможные потери $\left(-\sum_{l=k+1}^{n} \sum_{j=1}^{n} c_{i j}^{l}\left(p_{i j}^{l}\right)^{N}+\sum_{l=k+1}^{n} \sum_{i=1}^{n} c_{i j}^{l}\left(p_{i j}^{l}\right)^{N}\right) \quad$ от текущего выбора $c_{i j}^{k}$.

При этом на каждой итерации алгоритма происходит адаптация, пересчет вероятностей по следующим формулам:

$$
\begin{aligned}
& \left(p_{\mu \nu}^{l}\right)^{N+1}=\left(p_{\mu \nu}^{l}\right)^{N} q_{u}+p_{u}, \\
& \left(p_{i j}^{l}\right)^{N+1}=\left(p_{i j}^{l}\right)^{N} q_{u}, i, j=\overline{1, n}, i \neq \mu, j \neq v, \\
& \left(p_{i j}^{k}\right)^{N+1}=\left(p_{i j}^{k}\right)^{N}, i, j=\overline{1, n}, k=\overline{l+1, n,}
\end{aligned}
$$

где $p_{u}$ - настраиваемый параметр алгоритма, $q_{u}+p_{u}=1$.

Сам адаптивный алгоритм решения трехиндексной аксиальной задачи о назначениях (1)-(5) можно представить следующей схемой [8].

\section{Алгоритм.}

1. Задать начальное распределение вероятностей для каждого $k=\overline{1, n}\left(p_{i j}^{k}\right)^{1}=\frac{1}{n^{2}}$, $i, j=\overline{1, n}$.

Задать максимальное количество итераций $N_{\max }$, положить $N=1$.

2. Задать множества $I=\{1, \ldots, n\}$, $J=\{1, \ldots, n\}$, положить $k=1$.

3. Вычислить

$$
\begin{gathered}
\min _{i \in I, j \in J}\left\{c_{i j}^{k}-\left(\sum_{l=k+1}^{n} \sum_{j \in J}^{n} c_{i j}^{l}\left(p_{i j}^{l}\right)^{N}+\sum_{l=k+1}^{n} \sum_{i \in I}^{n} c_{i j}^{l}\left(p_{i j}^{l}\right)^{N}\right)\right\}= \\
=c_{\mu \nu}^{l}-\left(\sum_{l=k+1}^{n} \sum_{j \in J}^{n} c_{\mu j}^{l}\left(p_{\mu j}^{l}\right)^{N}+\sum_{l=k+1}^{n} \sum_{i \in I}^{n} c_{i v}^{l}\left(p_{i v}^{l}\right)^{N}\right),
\end{gathered}
$$

зафиксировать соответствующее назначение $\left(x_{\mu \nu}^{k}\right)^{N}=1$.

4. Пересчитать вероятности по формулам

$\left(p_{\mu \nu}^{l}\right)^{N+1}=\left(p_{\mu \nu}^{l}\right)^{N} q_{u}+p_{u}$,

$\left(p_{i j}^{l}\right)^{N+1}=\left(p_{i j}^{l}\right)^{N} q_{u}, i, j=\overline{1, n}, i \neq \mu, j \neq v$,

$\left(p_{i j}^{k}\right)^{N+1}=\left(p_{i j}^{k}\right)^{N}, i, j=\overline{1, n}, k=\overline{l+1, n}$.

5. Пересчитать безусловные вероятности по формуле

$$
\bar{p}^{N+1}=\frac{N}{N+1}\left(\bar{p}^{N}+\frac{1}{N} p^{N+1}\right) .
$$

\section{6. Изменить множества}

$I, J: I=I \backslash\{\mu\}, J=J \backslash\{v\}$.

7. Проверить: $k=n$ ? Если нет, то положить $k=k+1$ и переход к шагу 3 , если да, то переход к шагу 8.

8. Возможная смена рекорда $(X)^{N}$, $(L(X))^{N},(P)^{N}$.

9. Проверить $N=N_{\max }$ ? Если нет, то положить $N=N+1$ и перейти к шагу 2, если да, то записать ответ. В качестве ответа записывается последний рекорд.

Для анализа эффективности построенного адаптивного алгоритма был проведен вычислительный эксперимент, результаты которого также представлены в [8]. Один из выводов на основании эксперимента заключается в том, что на итоговый результат существенное влияние оказывает параметр $p_{u}$. Причем при разном количестве итераций и для матриц разной размерности наилучший результат достигается с разными значениями $p_{u}$ (рис. 1).

\section{Улучшение поисковых способностей алгоритма}

Описанные в предыдущем разделе (и более подробно в [8]) наблюдения приводят к идее изменения параметра $p_{u}$ на каждой итерации по некоторой заданной функции. Выбор функции, по которой изменяется $p_{u}$, основывается на следующих положениях:

- сначала осуществляется поиск в окрестности некоторого текущего решения для его улучшения;

- затем происходит выход из окрестности данного решения, с целью избежать «застревания» в локальном минимуме;

- после чего начинается поиск в окрестности другого решения.

Схематично данная идея представлена на рис. 2.

Для определения понятия окрестности решения $X^{N}$ аксиальной $3 \mathrm{OH}$ воспользуемся определением расстояния Хэмминга.

Определение 1. [6] Расстояние Хэмминга число позиций, в которых различаются соответствующие элементы двух матриц одинаковой размерности. 


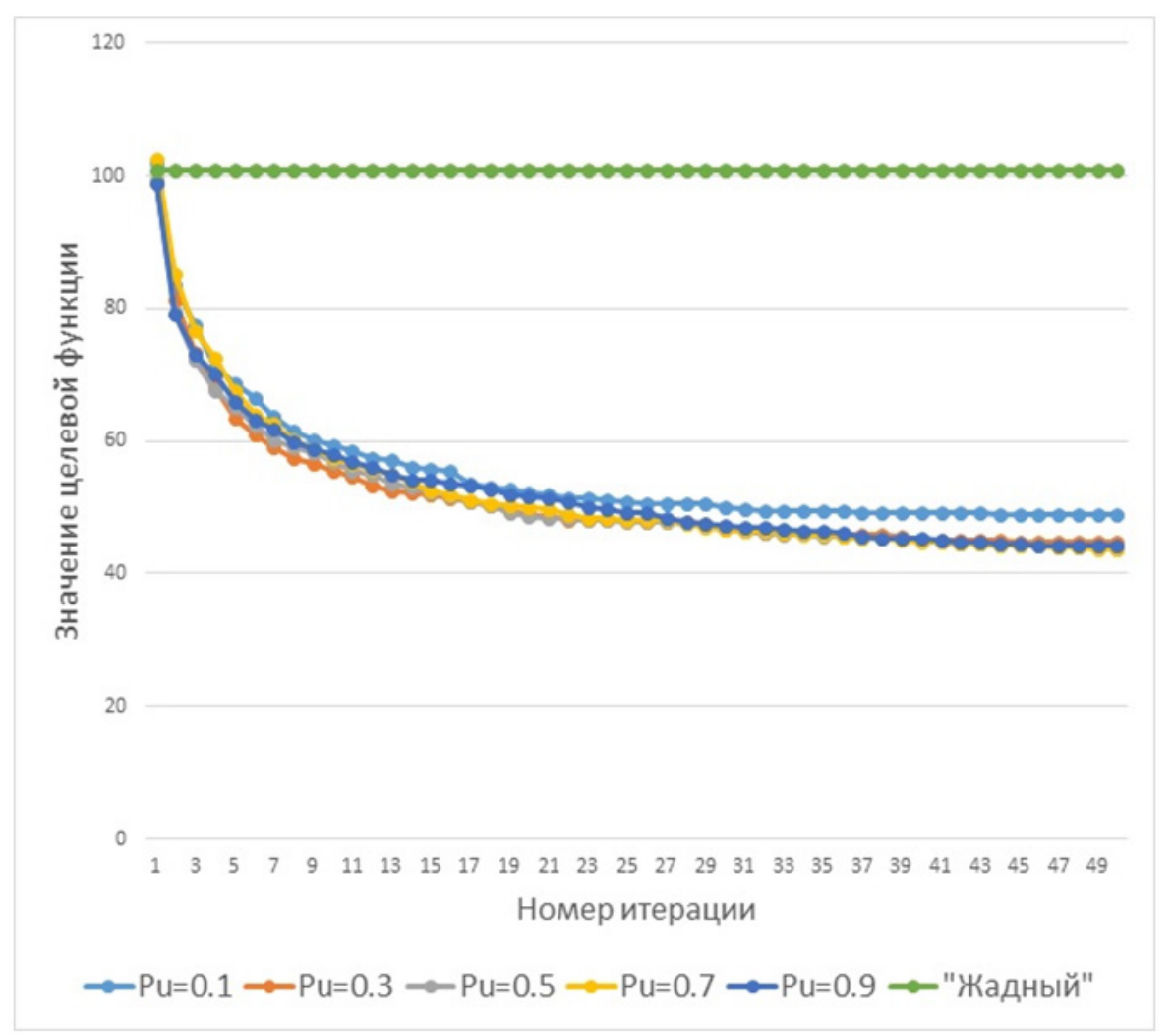

Рис. 1. Грабик работы алгоритма с разными параметрами $p_{u}$

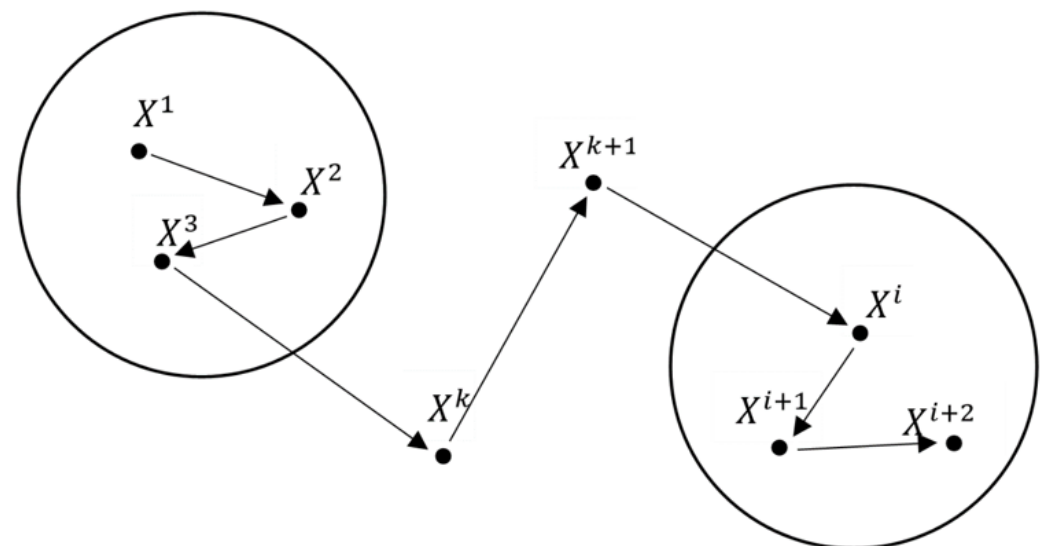

Рис. 2. Поиск решений в окрестности текущего и выход из локального минимума

Определение 2. $k$-окрестностью решения $X$ трехиндексной аксиальной $3 \mathrm{OH}$ назовем множество $\bar{\Omega}$ решений $\tilde{X}$, для которых выполняется неравенство $d(X, \tilde{X}) \leq k, \quad$ где $d(X, \tilde{X})$ - расстояние Хэмминга, $k \in \mathbb{Z}$.

Замечание 1. При $k=2 n$, окрестность допустимой точки трехиндексной аксиальной $3 \mathrm{OH}$ - есть все множество допустимых решений.

Основной вопрос, ответ на который можно получить экспериментально, заключается в том, каким именно образом следует изменять значения параметра $p_{u}$.
Значение параметра $\left(\pi_{\mathrm{i} j}^{k}\right)^{N+1}$ влияет на увеличение или уменьшение текущей вероятности $\left(p_{\mathrm{i} j}^{k}\right)^{N}$, представляя собой полный аналог направления движения в градиентном алгоритме. Величина $p_{u}$, в свою очередь, говорит о том, на сколько нужно изменить эту вероятность, что соответствует размеру шага.

Исходя из этого, рассмотрим две пограничные ситуации. Положим $p_{u}=0$. Тогда мы видим, что $\left(p_{\mathrm{i} j}^{k}\right)^{N+1}=\left(p_{\mathrm{i} j}^{k}\right)^{N}$ и пересчета вероятностей на $N+1$ шаге не произойдет. В обратном случае, положив $p_{u}=1,\left(p_{\mathrm{ij}}^{k}\right)^{N+1}$ ста- 
Вероятностный подход к решению трехиндексной аксиальной задачи о назначениях ...

новится равным $\left(\pi_{\mathrm{ij}}^{k}\right)^{N+1} \in\{0,1\}$ и алгоритм становится детерминированным.

Рассмотрим теперь значения $p_{u}$, лежащие в промежутке от 0 до 1 . Очевидно, что значения $p_{u}$, близкие к нулю, дадут незначительное изменение вероятности $\left(p_{\mathrm{i} j}^{k}\right)^{N+1}$, и, наоборот, близкие к единице значительно изменят вероятность $\left(p_{\mathrm{ij}}^{k}\right)^{N+1}$ в сторону ее детерминации до 1 или 0 [9].

Результаты вычислительного эксперимента (табл. 2) подтвердили, что при малых значениях параметра пересчета условных вероятностей $p_{u}$ (порядка $\frac{1}{n^{2}}$, где $n$-размерность матрицы) расстояние Хэмминга между решениями аксиальной $3 \mathrm{OH}$ мало (от итерации к итерации решение мало изменяется), в то время, как при значениях $p_{u}$, близких к 1 , расстояние Хэмминга стремится к $2 n$, т. е. решение практически полностью меняется.

Таким образом, идею изменения параметра $p_{u}$, описанную выше, можно переформулировать следующим образом:

- сначала берется малое значение параметра $p_{u}$, что позволяет осуществлять поиск в окрестности некоторого текущего решения для его улучшения;

- затем происходит увеличение $p_{u}$ с целью выхода из окрестности данного решения, дабы избежать «застревания» в локальном минимуме;

- после чего $p_{u}$ вновь уменьшается для того, чтобы попасть в окрестность другого решения.

\section{РЕЗУЛЬТАТЫ И ИХ ОБСУЖДЕНИЕ}

В табл. 1 приведены значения целевой функции аксиальной $3 \mathrm{OH}$ для матриц разной размерности. В ходе вычислительного эксперимента проводились серии по 100 испытаний для каждого случая. Матрицы заполнялись случайными числами из диапазона от 1 до 100.

В табл. 1 жирным шрифтом выделены несколько лучших значений при разных значениях параметра $p_{u}$.

В табл. 2 и на рис. 3 представлена зависимость расстояния Хэмминга от параметра $p_{u}$ для разных размерностей задачи.

Как показывают результаты тестов, расстояние Хэмминга напрямую зависит от параметра $p_{u}$ и от размерности задачи (при значениях параметра $p_{u}$ меньше $\frac{1}{n^{2}}$, где $n-$ размерность матрицы, расстояние Хэмминга стремится к нулю, при значениях, близких к 1, расстояние Хэмминга стремится к $2 n$ ).

Дальнейшая серия экспериментов была проведена с целью подтвердить или опровергнуть предположение, согласно которому изменение параметра пересчета вероятностей $p_{u}$ на каждой итерации по некоторой заданной функции должно привести к улучшению значения целевой функции.

В ходе эксперимента рассматривались следующие классы функций:

$$
f_{1}(i, a, b, c, d)=a e^{-b(i-c)^{2}}+d,
$$

где $a, b, c, d \in \mathbb{R}$ - заданные числа, $i \in \mathbb{Z}$ - номер итерации (рис. 4);

Таблица 1

Сравнение значений иелевой функиии в зависимости от параметра $p_{u}$ ичисла итераций

\begin{tabular}{|c|c|c|c|c|c|c|c|c|c|c|}
\hline \multirow{2}{*}{\multicolumn{2}{|c|}{$\frac{\text { Размерность }}{\text { Число итераций }}$}} & \multicolumn{3}{|c|}{$10 \times 10 \times 10$} & \multicolumn{3}{|c|}{$50 \times 50 \times 50$} & \multicolumn{3}{|c|}{$100 \times 100 \times 100$} \\
\hline & & 10 & 50 & 100 & 10 & 50 & 100 & 10 & 50 & 100 \\
\hline \multirow{8}{*}{  } & $p_{u}=0.001$ & 90 & 89 & 89 & 103 & 96 & 89 & 135 & 125 & 124 \\
\hline & $p_{u}=0.01$ & 78 & 67 & 64 & 85 & 74 & 71 & 132 & 119 & 117 \\
\hline & $p_{u}=0.1$ & 51 & 43 & 42 & 84 & 71 & 68 & 132 & 122 & 118 \\
\hline & $p_{u}=0.2$ & 49 & 41 & 39 & 86 & 74 & 69 & 135 & 123 & 120 \\
\hline & $p_{u}=0.3$ & 52 & 41 & 39 & 87 & 75 & 71 & 135 & 123 & 121 \\
\hline & $p_{u}=0.5$ & 53 & 40 & 38 & 89 & 78 & 73 & 137 & 124 & 123 \\
\hline & $p_{u}=0.7$ & 55 & 40 & 37 & 92 & 78 & 74 & 137 & 127 & 123 \\
\hline & $p_{u}=0.9$ & 56 & 39 & 37 & 95 & 79 & 74 & 138 & 127 & 122 \\
\hline
\end{tabular}


Таблица 2

Сравнение средних значений расстояния Хэмминга в зависимости от параметра $p_{u}$

\begin{tabular}{|c|c|c|c|c|}
\hline \multicolumn{2}{|c|}{ Размерность } & $10 \times 10 \times 10$ & $50 \times 50 \times 50$ & $100 \times 100 \times 100$ \\
\hline \multirow{12}{*}{ 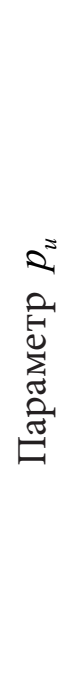 } & $p_{u}=0.0001$ & 0 & 2 & 6 \\
\hline & $p_{u}=0.0005$ & 0 & 5 & 69 \\
\hline & $p_{u}=0.001$ & 1 & 32 & 111 \\
\hline & $p_{u}=0.005$ & 1 & 56 & 135 \\
\hline & $p_{u}=0.01$ & 4 & 65 & 153 \\
\hline & $p_{u}=0.05$ & 7 & 79 & 172 \\
\hline & $p_{u}=0.1$ & 10 & 81 & 180 \\
\hline & $p_{u}=0.2$ & 13 & 84 & 183 \\
\hline & $p_{u}=0.3$ & 15 & 88 & 189 \\
\hline & $p_{u}=0.5$ & 15 & 90 & 192 \\
\hline & $p_{u}=0.7$ & 16 & 90 & 193 \\
\hline & $p_{u}=0.9$ & 17 & 93 & 195 \\
\hline
\end{tabular}

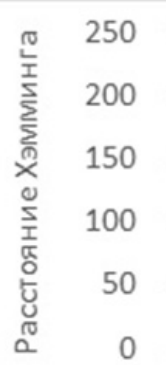

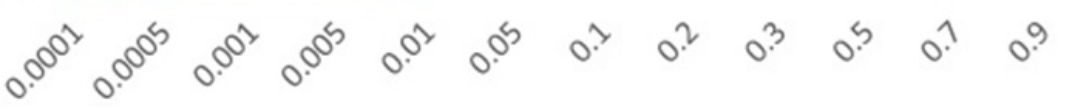

Значение параметра ри

$-10 \times 10 \times 10-50 \times 50 \times 50-100 \times 100 \times 100$

Рис. 3. Зависимость расстояния от параметра $p_{u}$

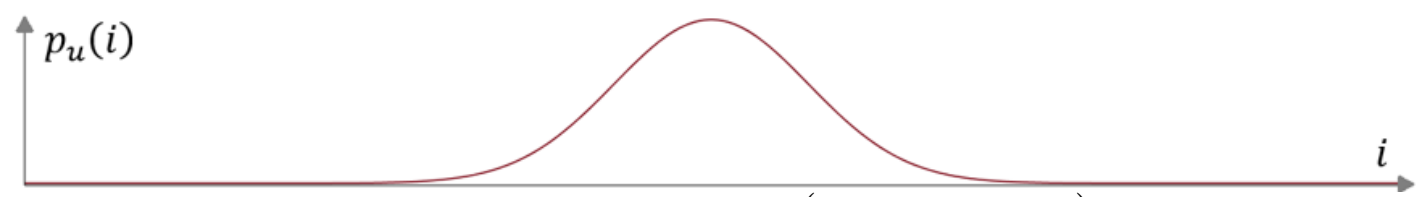

Рис. 4. График функиии $f_{1}(i, 0.2,0.2,25,0.1)$

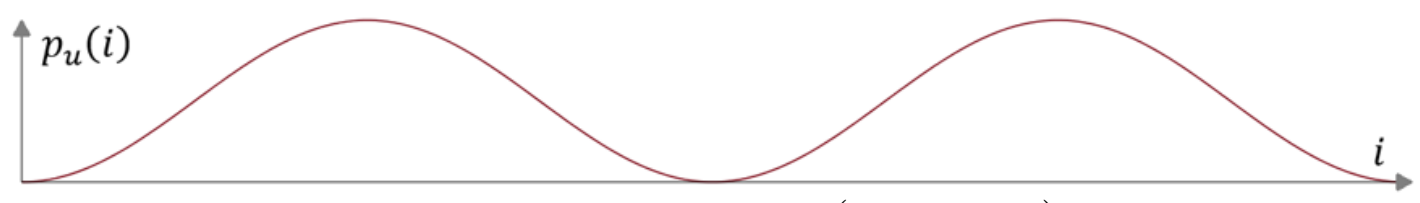

Рис. 5. График функиии $f_{2}(i, 0.1,0.25,0)$

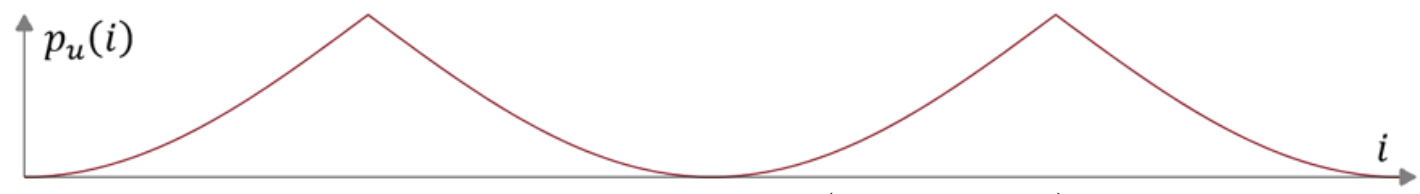

Рис. 6. График функиии $f_{3}(i, 0.1,0.25 \pi, 0)$ 
Вероятностный подход крешению трехиндексной аксиальной задачи о назначениях ...



Рис. 7. График функции $f_{4}(i, 0.05,0.1,0)$

Таблица 3

Сравнение значений иелевой функции в зависимости от функиии $p_{u}(i)$ и числа итераций для матрии разной размерности

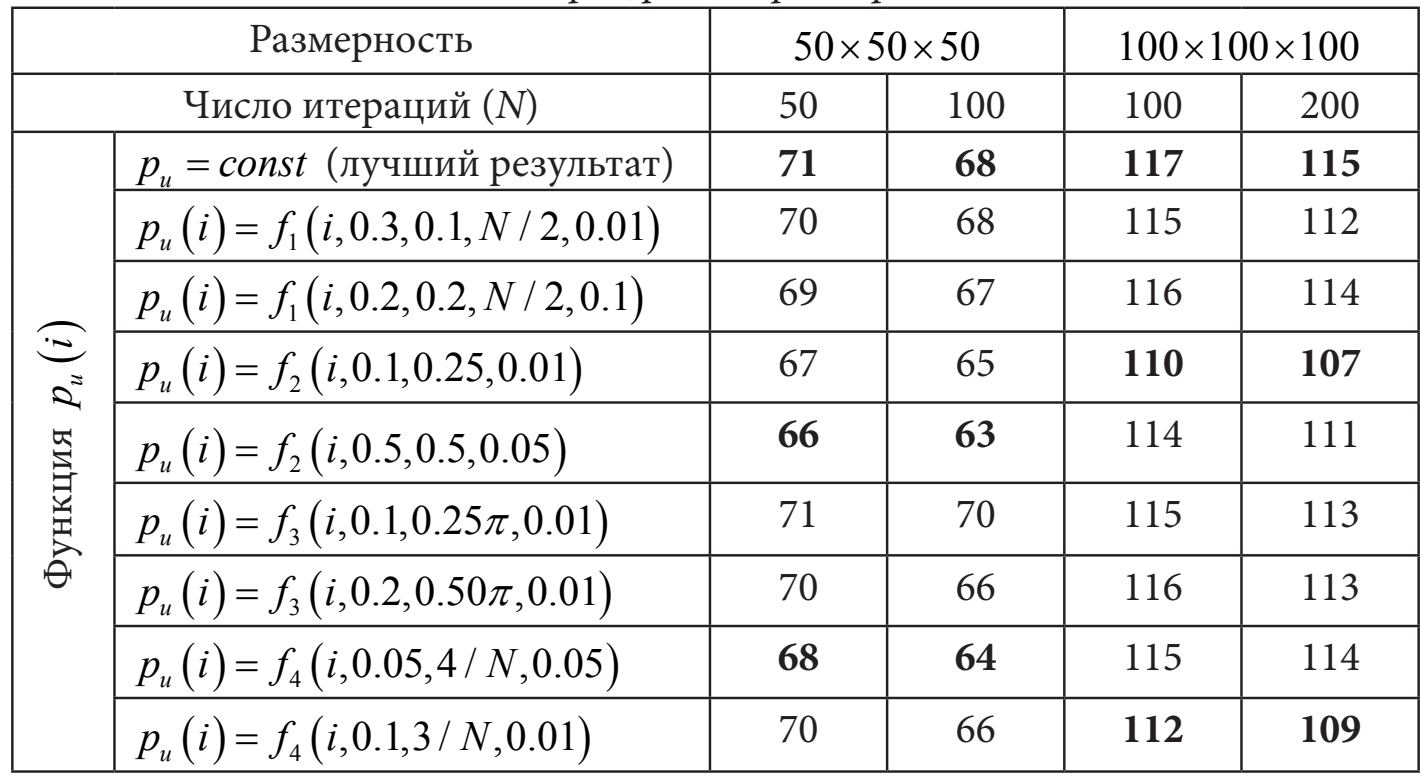

$$
f_{2}(i, a, b, c)=a(1-\cos (b x))+c,
$$

где $a, b \in \mathbb{R}$ - заданные числа, $i \in \mathbb{Z}$ - номер итерации (рис. 5);

$$
f_{3}(i, a, b, c)=a(1-|\cos (b x)|)+c,
$$

где $a, b \in \mathbb{R}$ - заданные числа, $i \in \mathbb{Z}$ - номер итерации (рис. 6);

$$
f_{4}(i, a, b, c)=a\left(1-\cos \left(e^{b x}\right)\right)+c,
$$

где $a, b \in \mathbb{R}$ - заданные числа, $i \in \mathbb{Z}$ - номер итерации (рис. 7);

В табл. 3 внесены значения целевой функции аксиальной $3 \mathrm{OH}$ при приведенных выше функциях $p_{u}(i)=f_{k}(i)$.

В табл. 3 жирным шрифтом выделены несколько лучших значений при различном изменении параметра $p_{u}$. Вычислительный эксперимент с $p_{u}=$ const показал, что наилучшие результаты достигаются в следующих ситуациях:

- для матриц размерности $50 \times 50 \times 50$ при $p_{u}=0.1$,

- для матриц размерности $100 \times 100 \times 100$ при $p_{u}=0.01$.
Как видно из табл. 3, использование функциональной зависимости параметра $p_{u}$ от числа итераций приводит к улучшению результатов, особенно на больших итерациях $(N>n)$. Однако для задач разных размерностей сами функции могут быть разными. Лучшие значения целевой функции аксиальной $3 \mathrm{OH}$ были показаны с использованием функциональных зависимостей $f_{2}(i, a, b, c)=a(1-\cos (b x))+c$ и $f_{4}(i, a, b, c)=a\left(1-\cos \left(e^{b x}\right)\right)+c$.

\section{ВЫВОДЫ}

1. В целом, предположение об улучшении значений целевой функции трехиндексной аксиальной $3 \mathrm{OH}$ при изменении $p_{u}$ по заданной функции подтвердилось.

2. Наилучший результат дают периодические функции, однако это улучшение проявляется при относительно большом количестве итераций $(N>n)$.

3. Для матриц разной размерности должны использоваться разнообразные функции пересчета параметра $p_{u}$. 


\section{ПЕРСПЕКТИВЫ ИССЛЕДОВАНИЯ}

На основании выше изложенного можно говорить, что дальнейшие исследования в области изучения адаптивного алгоритма могут быть направлены на поиск новых функций изменения параметра $p_{u}$. Например, функций, изменяющих $p_{u}$ пропорционально расстоянию Хэмминга между текущим и прошлым решениями, функций, зависящих от смены рекорда и т. п. Также отдельное исследование должно быть посвящено уменьшению времени работы алгоритма, например, с помощью составления специальной дополнительной матрицы, которая позволит избежать множественных вложенных обходов по кубической матрице затрат.

\section{СПИСОК ЛИТЕРАТУРЫ}

1. Афраймович, Л. Г. Многоиндексные транспортные задачи с декомпозиционной структурой / Л. Г. Афраймович // Автоматика и телемеханика. - 2012. - № 1. - С. 130-147.

2. Афраймович, Л. Г. Эвристический метод решения целочисленных декомпозиционных многоиндексных задач / Л. Г. Афраймович // Автоматика и телемеханика. - 2014. - № 8. C. 3-18.

3. Гимади, Э. Х. Аксиальные трехиндексные задачи о назначениях и коммивояжеpa: быстрые приближенные алгоритмы и их вероятностный анализ / Э. Х. Гимади, А. И. Сердюков // Изв. Вузов. Математика. 1999. - № 12. - C. 19-25.

4. Корбут, А. А. Дискретное программирование / А. А. Корбут, Ю. Ю. Финкельштейн [под ред. Д. Б. Юдина]. - М. : Наука, 1969. 368 c.
5. Львович, Я. Е. Конструирование адаптивных схем перебора для решения дискретных задач оптимизации / Я. Е. Львович, А. И Каплинский, Г. Д. Чернышова, О. И. Черных // В сб.: Актуальные проблемы фундаментальных наук. - М. : Изд-во МГТУ, 1991.

6. Малюгина, О. А. Задача комплектования штатов / О. А. Малюгина, С. Н. Медведев, Г. Д. Чернышова // Системное моделирование социально-экономических процессов : труды 31-й Международной научной школы-семинара, Воронеж, 1-5 октября 2008 г. : в 3 ч. Воронеж : ИПЦ Воронеж. гос. ун-т., 2008. Ч. III. - C. 265-272.

7. Медведев, С. Н. Адаптивные алгоритмы решения трехиндексных задач о назначениях / С. Н. Медведев // Современные методы прикладной математики, теории управления и компьютерных технологий: сб. тр. VI Междунар. конф. «ПМТУКТ-2013», Воронеж, 1016 сентября 2013 г. - Воронеж: ИПЦ Воронеж. гос. ун-т, 2013. - С. 153-156.

8. Трегубов, А. Г. Вероятностный подход к решению трехиндексной аксиальной задачи о назначениях / А. Г. Трегубов, С. Н. Медведев // Вестник Воронеж. гос. ун-та. Сер. Системный анализ и информационные технологии. 2015. - № 4. - 12 c.

9. Чернышова, Г. Д. Об использовании вероятностного аналога алгоритма покоординатного спуска в задаче о минимальном покрытии / Г. Д. Чернышова, С. В. Писковецкий - Воронеж: Воронеж. гос. ун-т., 2001. $14 \mathrm{c}$.

10. Crama, Y. Approximation algorithms for three-dimensional assignment problems with triangle inequalities / Y. Crama, F.C.R. Spieksma // European J. Oper. Res. - 1992. - Vol. 60. - P. 273279. 
Вероятностный подход к решению трехиндексной аксиальной задачи о назначениях ...

Трегубов А. Г. - аспирант кафедры вычисли- Tregubov A. G. - postgraduate of the Departтельной математики и прикладных информа- ment of Computing Mathematics and Applied ционных технологий факультета прикладной Information Technology, Applied Mathematics, математики, информатики и механики Воро- Informatics and Mechanics Faculty, Voronezh нежского государственного университета. State University.

E-mail: n.a.p.o.l.e.o.n@mail.ru

E-mail: n.a.p.o.l.e.o.n@mail.ru

Медведев С. Н. - кандидат физико-математических наук, доцент кафедры вычислительной математики и прикладных информационных технологий факультета прикладной математики, информатики и механики Воронежского государственного университета. E-mail: s_n_medvedev@mail.ru

Medvedev S. N. - Candidate of Physico-Mathematical Sciences, Lecturer of the Department of Computing Mathematics and Applied Information Technology, Applied Mathematics, Informatics and Mechanics Faculty, Voronezh State University.

E-mail: s_n_medvedev@mail.ru 